\title{
High-dose intramyocardial HMGB1 induces long-term cardioprotection in sheep with myocardial infarction
}

\author{
María del Rosario Bauzá ${ }^{1}$. Carlos Sebastián Giménez ${ }^{1}$ Paola Locatelli ${ }^{1}$ • Andrea De Lorenzi ${ }^{2}$ - Anna Hnatiuk ${ }^{1}$. \\ Maurizio C. Capogrossi ${ }^{3}$ - Alberto Crottogini ${ }^{1}$ • Luis Cuniberti ${ }^{1} \cdot$ Fernanda Daniela Olea $^{1}$ (i)
}

(C) Controlled Release Society 2019

\begin{abstract}
In rodents with acute myocardial infarction (AMI), high mobility group box 1 (HMGB1) injection has produced controversial results. Given the lack of data in large mammals, we searched the dose that would promote angiogenesis and expression of specific regenerative genes in sheep with AMI (protocol 1) and, subsequently, use this dose to study long-term effects on infarct size and left ventricular (LV) function (protocol 2). Protocol 1: Sheep with AMI received $250 \mu \mathrm{g}$ (high-dose, $n=7$ ), $25 \mu \mathrm{g}$ (lowdose, $n=7)$ HMGB1, or PBS (placebo, $n=7)$ in 10 intramyocardial injections $(0.2 \mathrm{ml} \mathrm{each})$ in the peri-infarct area. Seven days later, only the high-HMGB1-dose group exhibited higher microvascular densities, Ki67-positive cardiomyocytes, and overexpression of VEGF, Ckit, Tbx20, Nkx2.5, and Gata4. Protocol 2: Sheep with AMI received HMGB1 $250 \mu \mathrm{g}(n=6)$ or PBS $(n=6)$. At 60 days, HMGB1-treated sheep showed smaller infarcts ( $8.5 \pm 2.11$ vs. $12.2 \pm 1.97 \% \mathrm{LV}$ area, $P<0.05$, ANOVA-Bonferroni) and higher microvascular density (capillaries, $1798 \pm 252$ vs. $1266 \pm 250 / \mathrm{mm}^{2}$; arterioles, $18.3 \pm 3.9$ vs. $11.7 \pm 2.2 / \mathrm{mm}^{2}$; both $P<0.01)$. Echocardiographic LV ejection fraction, circumferential shortening, and wall thickening increased from day 3 to 60 with HMGB1 (all $P<0.05$ ). Conclusion: in ovine AMI, high-dose HMGB1 induces angio-arteriogenesis, reduces infarct size, and improves LV function at 2 months post-treatment.
\end{abstract}

Luis Cuniberti and Fernanda Daniela Olea contributed equally to this work.

\section{Key messages}

- HMGB1 reduces infarct size and preserves LV function in ovine myocardial infarction

- HMGB1 increases capillary and arteriolar density in the infarct border zone

- HMGB1 induces overexpression of angiogenic and cardiomyogenic genes

- HMGB1-treated sheep show 15-fold higher density of cycling cardiomyocytes vs. placebo

- High-dose HMGB1 induces long-term cardioprotection in a translational model of AMI

Electronic supplementary material The online version of this article (https://doi.org/10.1007/s13346-019-00628-z) contains supplementary material, which is available to authorized users.

Fernanda Daniela Olea

dolea@favaloro.edu.ar

1 Instituto de Medicina Traslacional, Trasplante y Bioingeniería (IMETTYB), Universidad Favaloro-CONICET, Solís 453, C1078AAI Buenos Aires, Argentina

2 Hospital Universitario de la Fundación Favaloro, Buenos Aires, Argentina

3 Division of Cardiology, Johns Hopkins Bayview Medical Center, Baltimore, MD, USA
Keywords HMGB1 · Cardioprotection · Acute myocardial infarction $\cdot$ Sheep

\section{Introduction}

Cardiovascular disease causes $31 \%$ of all deaths worldwide. In 2015, 46\% (approximately 9 million) of these deaths were due to ischemic heart disease, making this condition the leading cause of death in the world [1]. Its most severe complication is acute myocardial infarction (AMI) which leads to left ventricular (LV) remodeling, a process characterized by progressive replacement of the surviving contractile tissue by fibrosis, and heart failure [2]. Since the extent and progression rate of remodeling are largely dependent on infarct size, limiting the mass of necrotic myocardium is a key therapeutic goal [3].

Significant progress in this regard has been achieved through invasive revascularization procedures. However, factors like adverse socioeconomic condition, low educational level, and geographic barriers hinder equal access to these treatments $[4,5]$. Consequently, alternative strategies aimed at limiting infarct size are being intensively investigated. 
One of these is the injection of proteins (or their encoding genes) displaying angiogenic, cardiomyogenic, antifibrotic, and antiapoptotic effects [6,7].

Several growth factors and angiogenic cytokines have been tested in animal models of ischemic heart disease. Moreover, some of them like VEGF, FGF, and GM-CSF have been used in clinical trials [8].

High mobility group box 1 (HMGB1) is a non-histone chromatin-binding protein present in the nucleus of all cells, and displaying intracellular and extracellular actions. Within the cell, HMGB1 regulates transcription, DNA replication, DNA repair, and nucleosome structure $[9,10]$. When present in the extracellular space, HMGB1 has inflammatory effects and stimulates angiogenesis along with proliferation and differentiation of vascular and cardiac stem cells [11]. These features make HMGB1 a potentially useful target in cardiac diseases.

Whether HMGB1 in ischemic heart disease is beneficial or deleterious is a matter of controversy. In transgenic mice overexpressing HMGB1, Kitahara et al. showed that coronary artery ligation resulted in smaller infarcts and higher survival rate than in their non-transgenic littermates [12]. In mice with chronic heart failure HMGB1 enhanced LV function and attenuated LV remodeling [13]. Similar results were reported in rats with AMI [14, 15]. Of particular interest are reports showing a cardiomyogenic effect of HMGB1, elicited by recruitment, activation, and differentiation of cardiac progenitor cells $[13,16]$. This suggests that HMGB1 may be potentially useful not only in myocardial infarction, but also in other diseases characterized by loss of contractile tissue.

On the other hand, in a murine model of AMI, Qi et al. found that HMGB1 inhibition by angiotensin-converting enzyme 2 reduced infarct size by over $50 \%$ and preserved cardiac function [17]. Furthermore, Zhang et al. recently reported that the antiretroviral compound atazanavir attenuated postinfarct fibrosis and ventricular remodeling in rats, and that these effects were attributable to blocking of myocardial inflammatory cascades through an HMGB1/TLR 9 signaling pathway [18].

To our knowledge, all studies addressing the effect of HMGB1 in ischemic heart disease have been performed in mice and rats. In order to facilitate clinical translation of experimental results, data from large mammalian models more closely resembling to the human than laboratory rodents are needed. These include not only the effects of HMGB1 in the long term, but also the mechanisms leading to them and the appropriate dosage to be applied.

Hence, in the present study, we assessed the effects of intramyocardial HMGB1 on infarct size and LV function in sheep with acute AMI at 60 days after treatment and the possible mechanisms involved. The dose used was previously determined in a dose-response study on the same animal model.

\section{Materials and methods}

\section{Dose-response protocol}

\section{Animal model and treatment}

Twenty one adult Corriedale sheep of either sex, aged 24 36 months, and weighting $43 \pm 6 \mathrm{~kg}$ were premedicated with acepromazine maleate $(0.3 \mathrm{mg} / \mathrm{kg})$. Anesthesia was induced with intravenous propofol $3 \mathrm{mg} / \mathrm{kg}$ and maintained with $2 \%$ isoflurane in oxygen under mechanical ventilation (Neumovent, Córdoba, Argentina). A sterile left thoracotomy in the fourth intercostal space was performed under electrocardiographic and oxymetric monitoring (Edan patient EDAN Instrument Inc., Shenzhen, China).

Diagonal branches of the anterior descending coronary artery (LAD) were ligated to produce an anteroapical infarct of approximately $10-15 \%$ of the LV mass. The first diagonal branch was always avoided. Given that in sheep the LAD distribution displays inter-individual heterogeneity [19], the branches to be ligated were selected by visual inspection of the epicardial LAD anatomy.

To reduce the incidence of ventricular arrhythmias, we administered lidocaine (three bolus injections of $2 \mathrm{mg}$ each and a $2 \mathrm{mg} / \mathrm{kg} / \mathrm{h}$ infusion), amiodarone ( $150 \mathrm{mg}$ infusion in $2 \mathrm{~h}$ ), and atenolol (2 mg).

Four hours after ligature, animals were randomized to receive 10 intramyocardial injections of $0.2 \mathrm{ml}$ in the infarct border zone containing $125 \mu \mathrm{g} / \mathrm{ml}(250 \mu \mathrm{g}$ in total $)$ of HMGB1 (high-dose group, $n=7), 12.5 \mu \mathrm{g} / \mathrm{ml}(25 \mu \mathrm{g}$ in total) of HMGB1 (low-dose group, $n=7$ ), or vehicle (PBS, placebo group, $n=7$ ) using a $25-G$ needle. Injections were performed in the normoperfused myocardium surrounding the ischemic area within $5 \mathrm{~mm}$ of the infarct border, which was readily visible, and the needle was retained in the myocardium for $20 \mathrm{~s}$ after each injection to minimize reflux induced by systolic contraction. Taking into account this time and the time elapsed between injections ( $\sim 10 \mathrm{~s}$ ), the total treatment procedure lasted approximately $5 \mathrm{~min}$. Given the size of the ovine heart and the extent of the infarction, the number of injectates is appropriate to surround the infarct, and has been used by us in previous studies $[20,21]$. Then, the thoracotomy was closed and after removal of the tracheal tube, cephalotin $1 \mathrm{~g}$ i.v. was injected and sheep were returned to the animal house under analgesic treatment (nalbuphine $0.3 \mathrm{mg} / \mathrm{kg}$, subcutaneous). For both protocols, the nature of the injectates was blinded to all investigators until the end of data processing.

Seven days after AMI, the animals were euthanized with an overdose of propofol followed by an intravenous bolus of potassium chloride, and tissue samples of the injected infarct border zone were harvested, fixed in $10 \%$ formaldehyde, and embedded in paraffin for immunohistochemistry or frozen in 
liquid nitrogen and conserved at $-70{ }^{\circ} \mathrm{C}$ for RT-qPCR analysis. HMGB1 protein was kindly provided by HMGBiotech (Milan, Italy).

\section{Gene expression}

Frozen heart tissues were pulverized and RNA was extracted with Trizol reagent (Life Technologies Inc., Gaithersburg, MD, USA) according to the manufacturer's instructions. RNA was treated with DNase I (RQ1 RNase-Free DNase, Promega, Madison, WI, USA) and reverse transcription (High Capacity cDNA Reverse Transcription Kit (Applied Biosystems, Foster City, CA, USA) was performed). Realtime PCR (SYBR ${ }^{\circledR}$ Select Master Mix, Applied Biosystems) was performed using sheep-specific primers designed to quantify gene expression of VEGF, Tbx20, Ckit, $N k x 2.5$, and Gata4. To quantify and evaluate the RNA integrity, the constitutive GAPDH gene was amplified in each reaction (see all primers in Supplemental material). For each gene, a calibration curve was performed.

\section{Histology}

Tissue sample sections were deparaffinized and antigen retrieval was performed using citrate $(0.1 \mathrm{M}$; pH 6). Endogenous peroxidase was blocked with $3 \% \mathrm{H}_{2} \mathrm{O}_{2}$, and the slides were incubated during $1 \mathrm{~h}$ with a specific monoclonal antibody against smooth muscle actin (BioGenex, Fremont, CA, USA) to identify arterioles (vessels measuring 8-100 $\mu \mathrm{m}$ in diameter with a smooth muscle layer), or with the endothelial marker Biotinylated Euonymus Europaeus Lectin (Vector Laboratories, Burlingame, CA, USA) to identify capillaries (vessels measuring up to $10 \mu \mathrm{m}$ in diameter with only an endothelial wall). Biotin/streptavidin peroxidase was used as detection system and DAB chromogen as marker. Twenty matching fields from each slide $(\times 20$ magnification for arteriole and $\times 40$ for capillary quantification) were examined (Image-Pro Plus 4.1, Media Cybernetics, Silver Spring, MD, USA) and vascular density was expressed as number of arterioles or capillaries per $\mathrm{mm}^{2}$.

In order to search for cycling cardiomyocytes, tissue sections were incubated with a monoclonal antibody against the Ki67 antigen (Leica Biosystems, Newcastle, UK), posttreated with a biotinylated anti-mouse immunoglobulin antiserum (BioGenex, San Ramon, CA, USA) and revealed with DAB as chromogen. Subsequently, sections were incubated with an anti-sarcomeric $\alpha$-actin antibody (Dako, Carpinteria, CA, USA) and post-treated with the biotinylated antiserum followed by peroxidase substrate (ImmPACT VIP, Vector Laboratories). All slices undergoing immunohistochemistry were stained with hematoxylin.

\section{Therapeutic effects protocol}

\section{Surgery and treatment}

Twelve sheep were operated as described previously and treated with the dose found to be effective in the dose-response study, i.e., 10 intramyocardial injections of $0.2 \mathrm{ml}$ each in the infarct border zone containing $125 \mu \mathrm{g} / \mathrm{ml}$ (total dose $250 \mu \mathrm{g}$ in $2 \mathrm{ml}$ ) of HMGB1 (HMGB1 group, $n=6$ ) or PBS (placebo group, $n=6) 4 \mathrm{~h}$ after coronary occlusion, using the injection protocol explained above.

\section{Echocardiography}

At 3 days prior to coronary occlusion (baseline), 3, 30, and 60 days post-AMI, bidimensional echocardiography was performed (Sonos 5500, Hewlett-Packard, Palo Alto, CA, USA) under light sedation (diazepam $10 \mathrm{mg}$ i.v.) and with the animal lying on its right lateral decubitus. Parasternal long- and shortaxis views were recorded. Percent anterolateral wall thickening (\%AWTh), septal wall thickening (\%SWTh), circumferential shortening fraction (\%CSF), and ejection fraction $(\% \mathrm{EF})$ were measured. We have previously shown that echocardiographic parameters of systolic and diastolic LV performance in young adult sheep can be reliably extrapolated to the adult human [22], indicating that at least in this animal model echocardiography is useful to assess LV function.

\section{Infarct size}

At 60 days, the animals were euthanized and the heart was removed to measure infarct size. The LV was opened through an incision parallel to the posterior interventricular sulcus and extended flat before fixation. A photograph of the LV endocardial aspect was taken in order to quantify the infarcted area by morphometry. For this purpose, infarct size was quantified manually as a percentage of the total area of the LV (Image-Pro Plus 4.1).

\section{Cardiomyogenesis and gene expression}

At 60 days after treatment, tissue samples of the infarct border zone underwent immunohistochemistry to detect Ki67positive myocytes and real-time PCR to measure expression of VEGF, Tbx20, Ckit, Nkx2.5, and Gata4, following the same protocols described above.

\section{Statistical analysis}

Data were analyzed using one-way (vascular densities) or twoway (echocardiography parameters) ANOVA-Bonferroni. Student's $t$ test was applied to analyze infarct size. Results are expressed as mean \pm standard deviation. Values of $P<0.05$ were considered indicative of statistically significant differences. 


\section{Results}

\section{Dose-response study}

\section{Microvascular proliferation}

Microvascular densities at 7 days post-treatment are shown in Fig. 1. Capillary density increased only in the high-dose group $\left(2828 \pm 511\right.$ capillaries $\left./ \mathrm{mm}^{2}\right)$ with respect to placebo $(1711 \pm$ 194, $P<0.01$, ANOVA-Bonferroni) but not in low-dose animals $(2341 \pm 379, P=\mathrm{NS})$. Arteriolar density was almost identical for both doses of HMGB1 (high dose 39.4 \pm 11 ; low dose $39 \pm 14$ arterioles $/ \mathrm{mm}^{2}$ ). However, given the lower data scatter, only high-dose HMGB1 achieved statistically significant differences with regard to placebo (23.2 \pm 4 arterioles $\left./ \mathrm{mm}^{2}, P<0.05\right)$.

\section{Cardiomyogenesis}

The high HMGB1 dose enhanced the number of Ki67positive myocytes vs. placebo, $(4.6 \pm 1.1$ cardiomyocytes/ $\mathrm{mm}^{2}$ vs. $0.3 \pm 0.6, P<0.05$ ) (Fig. 2). The low HMGB1 dose $\mathbf{a}$

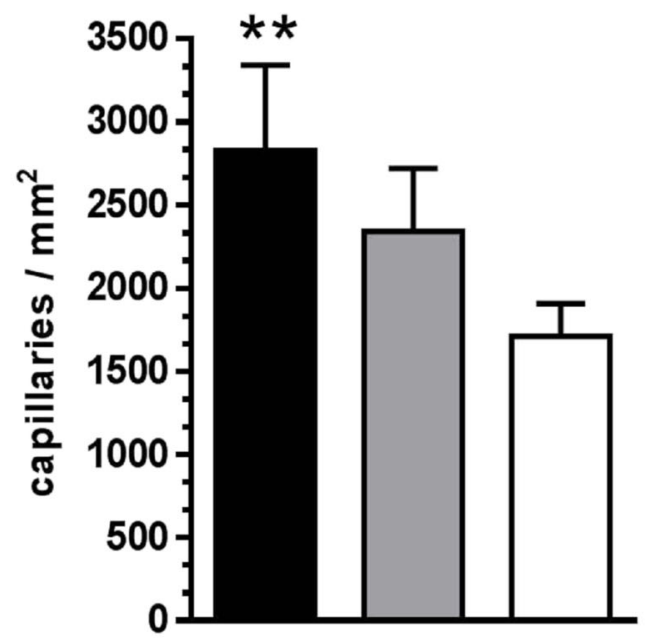

b

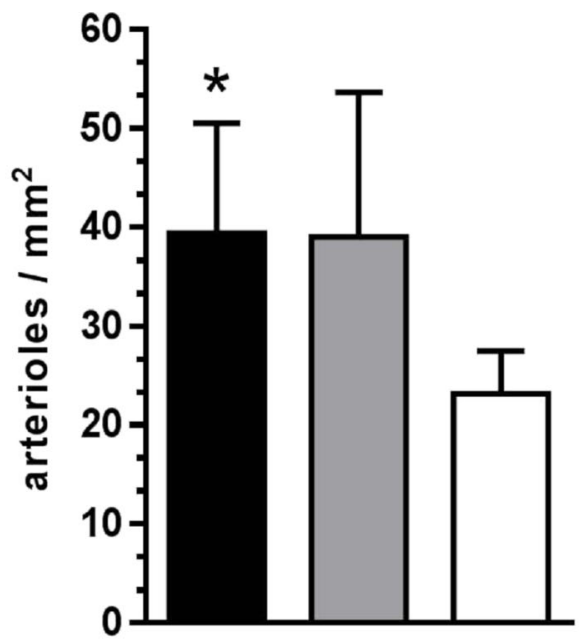

\section{$\square$ High dose $\square$ Low dose $\square$ placebo}

c

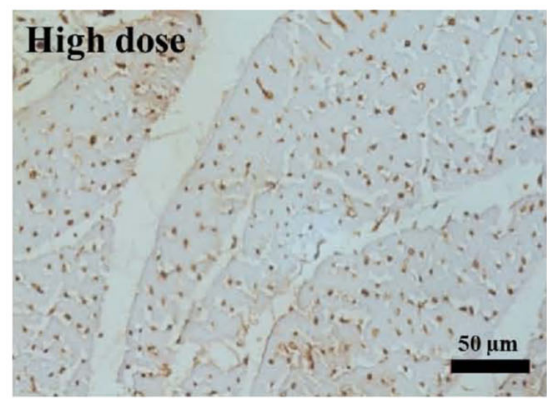

d

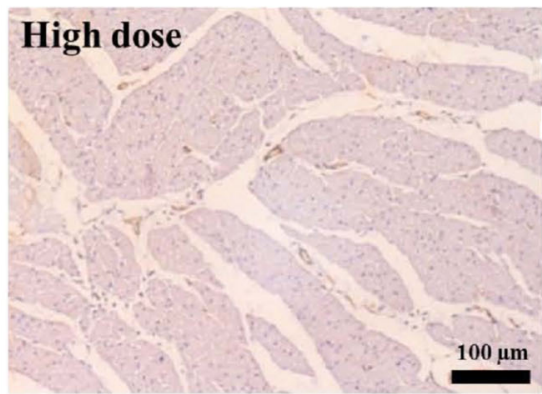

Fig. 1 Microvascular density at 7 days post-AMI. Arteriolar (a) and capillary (b) densities were higher than placebo with both doses of HMGB1, but only with the high dose the difference was statistically significant. $\mathbf{c}$ Representative slices of the injected peri-infarct region for each group showing capillaries stained with a biotinylated lectin (bars =
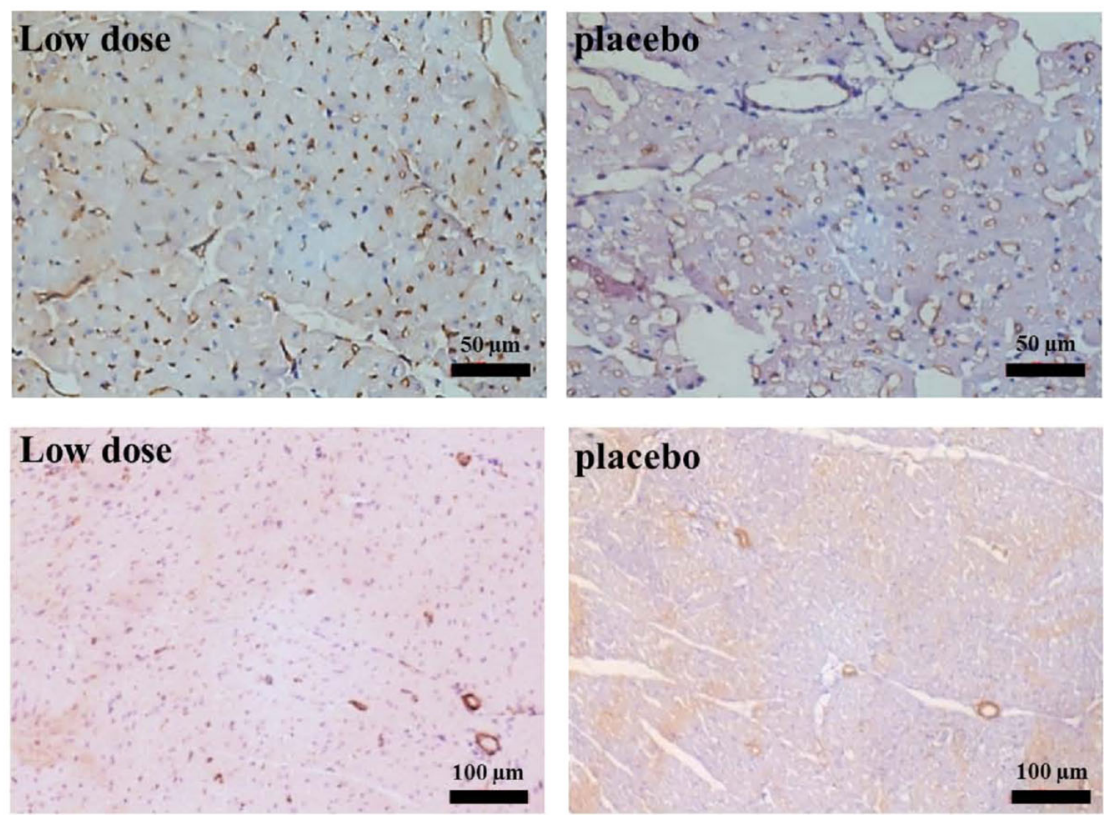

$50 \mu \mathrm{m})$. d Representative slices of the injected peri-infarct region for each group showing arterioles stained with an antibody against smooth muscle actin (bars $=100 \mu \mathrm{m}$ ). Results are means $\pm \mathrm{SD}$. $* P<0.05$ vs. placebo; $* * P<0.01$ vs. placebo 


\section{a}

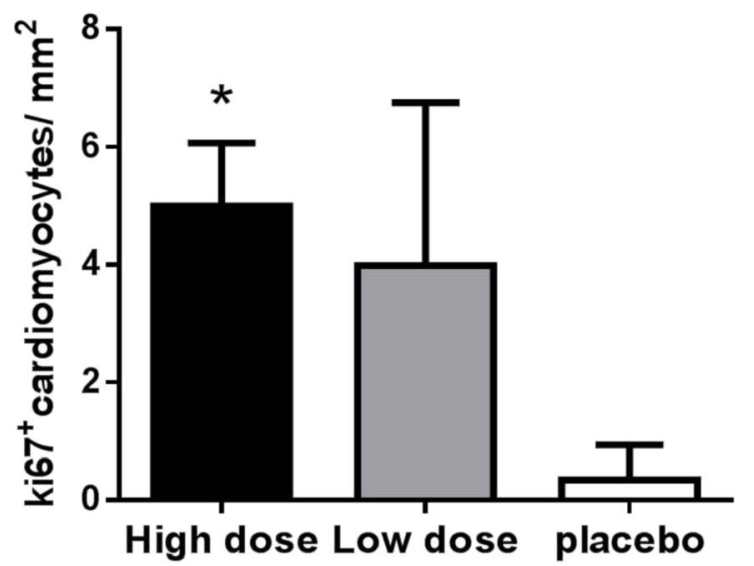

b

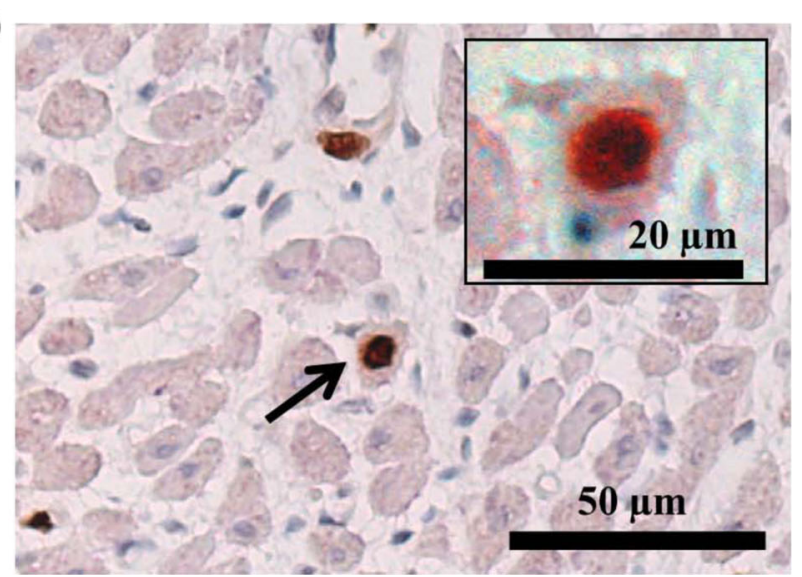

Fig. 2 Cycling cardiomyocytes at 7 days post-AMI. a The density of Ki67-positive cardiomyocytes was significantly higher in the high-dose HMGB1 group. b Representative image of high-dose HMGB1 group labeled by nuclear Ki67-positive (brown) and sarcomeric actin (violet) ( $\mathrm{bar}=50 \mu \mathrm{m})$. Arrow indicates mitotic figure in adult cardiomyocyte, magnified in the inset $($ bar $=20 \mu \mathrm{m})$. Results are means \pm SD. $* P<0.05$ vs. placebo

increased the number of Ki67-positive cells vs. placebo (4.0 \pm 2.8) but the difference did not achieve statistical significance.

\section{Gene expression}

Expression of VEGF Tbx20, Ckit, Nkx2.5, and Gata4 was significantly higher than placebo in the high-HMGB1-dose group. Gata4 expression was also higher than placebo in the low-HMGB1-dose group (Fig. 3).

\section{Therapeutic effects study}

On the basis of the dose-response protocol, we selected the high-dose HMGB1 to study its long-term effects on infarct size and LV function in the same animal model. We also assessed if the angiogenic, cardiomyogenic, and gene expression response observed at 7 days posttreatment was preserved at later time points.

\section{Infarct size}

At 60 days post-treatment, infarct size (as percent of LV area) was significantly lower in the HMGB1 group $(8.5 \pm 2.1 \%)$ than in the placebo group $(12.2 \pm 2 \%, P<0.05)$ (Fig. 4).

\section{LV function}

Table 1 shows LV function indexes prior to coronary occlusion (baseline, 3, 30, and 60 days post-AMI). At baseline, no differences between groups were found in $\% \mathrm{EF}$, $\%$ AWTh, \%SWTh, and \%CSF. As expected, all 4 parameters worsened significantly at 3 days post-occlusion vs. baseline in both groups. Both \%AWTh and \%SWTh improved significantly over time in HMGB1-treated animals, achieving at 60 days values that were not significantly different from baseline. This was not the case for placebo animals, in which wall thickening values were persistently depressed at day 60 of follow-up. On the other hand, $\% \mathrm{CSF}$ improved at 30 days as compared to 3 days in both groups. However, only the HMGB1 group preserved this improvement until the 60-day time point. Finally, \%EF achieved similar values in both groups at 30 days. However, as compared with the values at day 3 post-AMI, $\%$ EF improved significantly in HMGB1-treated animals.

\section{Microvascular proliferation}

Microvascular densities at 60 days post-treatment are shown in Fig. 5. Both arteriolar and capillary densities were significantly enhanced in the HMGB1 group with respect to placebo (arteriolar density HMGB1, $18.3 \pm 3.9$ arterioles $/ \mathrm{mm}^{2}$; placebo, $11.7 \pm 2.2, P<0.005$; capillary density HMGB1, $1798 \pm$ 252 capillaries $/ \mathrm{mm}^{2}$; placebo, $\left.1266 \pm 250, P<0.005\right)$.

\section{Cardiomyogenesis and gene expression}

At 60 days post-treatment, no Ki67-positive cardiomyocytes were observed in any groups. No differences were observed in the expression of VEGF, Tbx20, Ckit, Nkx2.5, and Gata4 between groups (Table 2).

\section{Discussion}

The effects of HMGB1 administration on infarct size and cardiac function have been investigated in mice and rats [13-15]. To the best of our knowledge, the present study is the first to evaluate the effects of HMGB1 in a large mammalian model of AMI. 


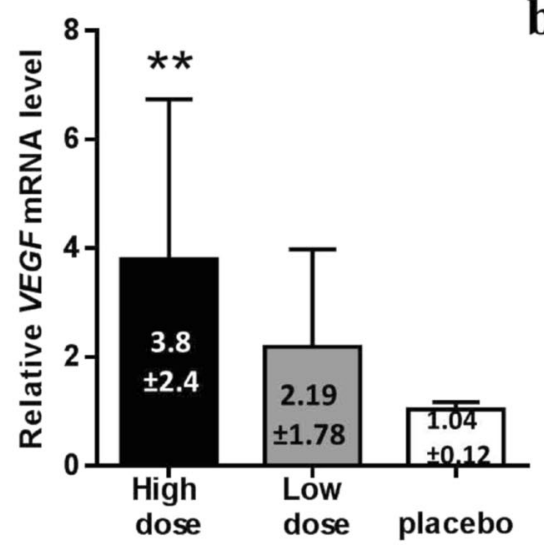

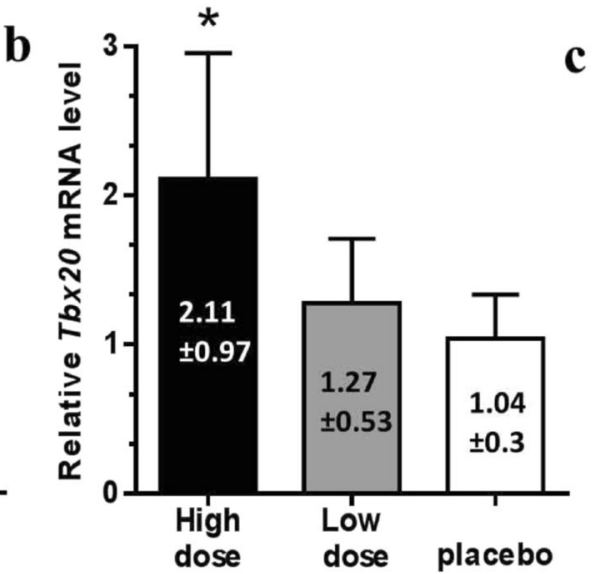

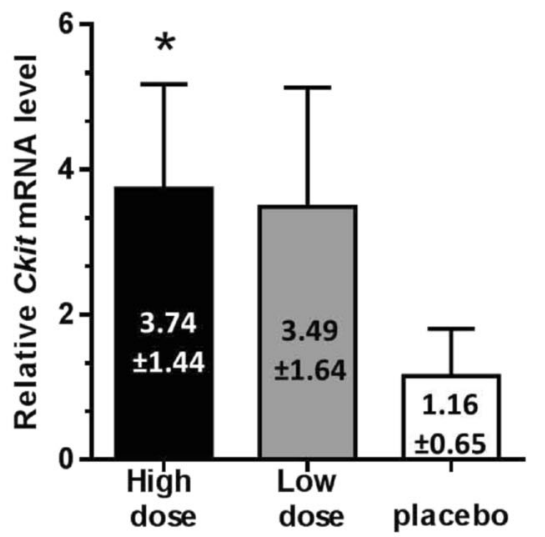

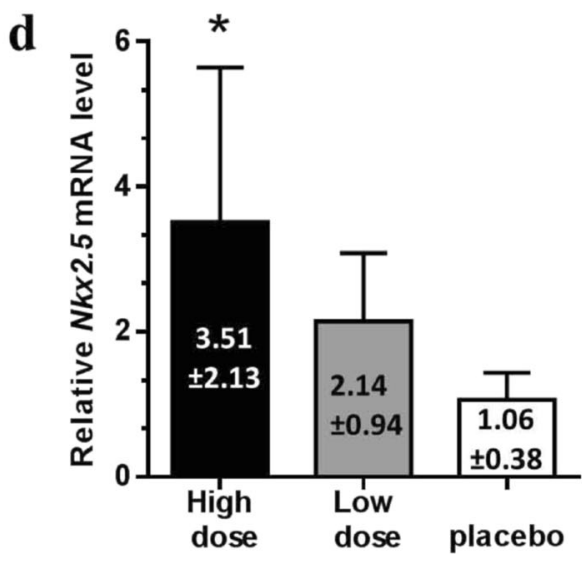

Fig. 3 mRNA relative expression of $\operatorname{VEGF}$ (a), Tbx20 (b), Ckit (c), $N k x 2.5$ (d), and Gata4 (e) at 7 days post-treatment. Sheep were injected with high-dose $(250 \mu \mathrm{g})$, low-dose $(25 \mu \mathrm{g}) \mathrm{HMGB} 1$ or placebo (PBS) $4 \mathrm{~h}$

When studying infarct size limitation in large mammals, pigs or sheep are usually used. We chose sheep because, unlike pigs, whose cardiomyocytes have up to 32 nuclei $[23,24]$, ovine cardiomyocytes have only $1-4$ nuclei [24], thus being more similar to the human. Another reason relates to the growth rate of pigs in long-term follow-up protocols. While adult sheep weighing $\sim 45 \mathrm{~kg}$ (as those used in

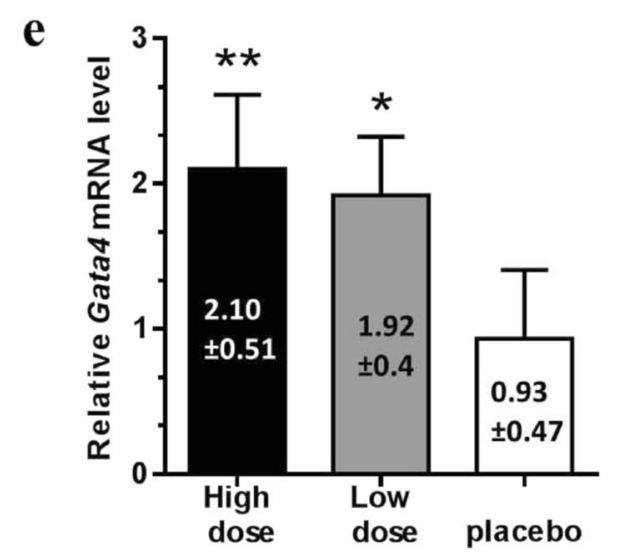

after coronary occlusion. Results are means $\pm \mathrm{SD}$. $* P<0.05$ vs. placebo; $* * P<0.01$ vs. placebo

our study) may increase their weight by $\sim 5 \%$ in 60 days, pigs may duplicate their body mass which, in turn, can complicate the interpretation of data concerning infarct size and ventricular remodeling. In addition, under our experimental conditions, during myocardial ischemia, pigs are more prone to develop irreversible ventricular fibrillation than sheep, leading to higher mortality.

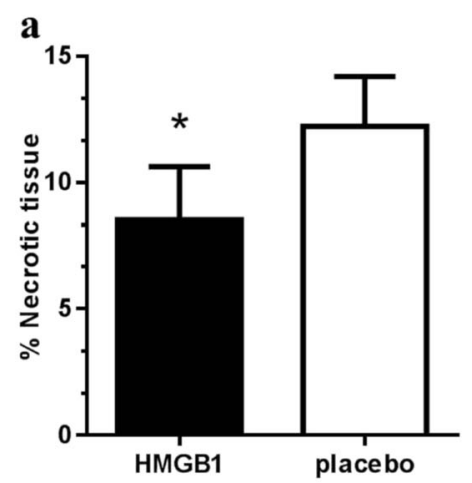

b

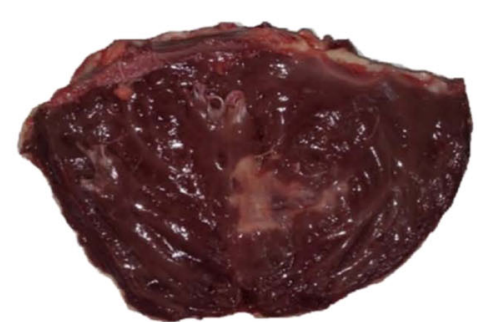

HMGB1

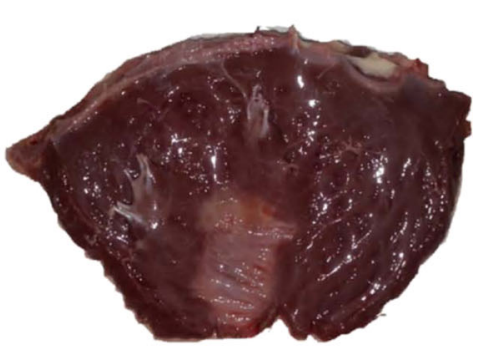

placebo

Fig. 4 Infarct size at 60 days post-AMI. a Infarct size as percent left ventricular area was significantly lower in the high-dose HMGB1 group. b Representative images of infarcted left ventricles in each group 
Table 1 Echocardiographic parameters at baseline, and 3, 30, and 60 days post-AMI

\begin{tabular}{llllll}
\hline Parameter & Treatment & Baseline & 3 days & 30 days & 60 days \\
\hline \%AWTh & HMGB1 & $48.7 \pm 9.3$ & $11.5 \pm 3.2^{*}$ & $27.3 \pm 12.4^{*}$ & $35.3 \pm 8.3 \#$ \\
& Placebo & $45.3 \pm 9.6$ & $18.1 \pm 8.9^{*}$ & $15 \pm 11.3^{*}$ & $25.2 \pm 14.7^{*}$ \\
$\%$ SWTh & HMGB1 & $42.2 \pm 14$ & $10.5 \pm 8.9^{*}$ & $30.9 \pm 16.7 \#$ & $27.6 \pm 16$ \\
& Placebo & $42 \pm 6.4$ & $12.8 \pm 9.7^{*}$ & $10.1 \pm 13.1^{*}$ & $21.2 \pm 10.7^{*}$ \\
$\%$ CSF & HMGB1 & $44.2 \pm 5.2$ & $29.7 \pm 6.7^{*}$ & $35.9 \pm 9.1$ & $38.4 \pm 9.1$ \\
& Placebo & $47.5 \pm 8.3$ & $26.5 \pm 8.3 *$ & $42.2 \pm 5.8 \#$ & $33.7 \pm 6^{*}$ \\
EDF & HMGB1 & $66.8 \pm 7.7$ & $39.8 \pm 8.6^{*}$ & $54.4 \pm 13.2 \#$ & $54.4 \pm 6.9 \#$ \\
& Placebo & $66 \pm 12.8$ & $43.4 \pm 5.6^{*}$ & $53.3 \pm 13.2$ & $56.9 \pm 8.9$ \\
ESD & HMGB1 & $41.1 \pm 3.2$ & $36.5 \pm 2.2$ & $40.8 \pm 1.3$ & $39.2 \pm 3.6$ \\
& Placebo & $41.5 \pm 4.0$ & $39.2 \pm 2.3$ & $38.9 \pm 4.9$ & $39.8 \pm 5.0$ \\
& HMGB1 & $24.5 \pm 3.1$ & $27.7 \pm 2.3$ & $28.9 \pm 3.7$ & $28.2 \pm 2.0$ \\
\hline
\end{tabular}

$\% A W T h$, percent anterior wall thickening; \%SWTh, percent septal wall thickening; \%CSF, percent circumferential shortening fraction; $\% E F$, percent ejection fraction; $E D D$, end-diastolic diameter; $E S D$, end-systolic diameter; $H M G B 1$, high mobility group box 1 protein. $* P<0.05$ vs. baseline; $\# P<0.05$ vs. 3 days. All data are means \pm SD
We injected the treatments $4 \mathrm{~h}$ after coronary ligation because the complex inflammatory response that occurs over the first few hours after acute myocardial infarction makes the myocardium especially unstable and vulnerable [25]. In fact, despite preventive antiarrhythmic treatment was administered in all animals (see Material and Methods), ventricular premature beats, bigeminy, and/or bursts of ventricular tachycardia occurred consistently early after occlusion. Furthermore,

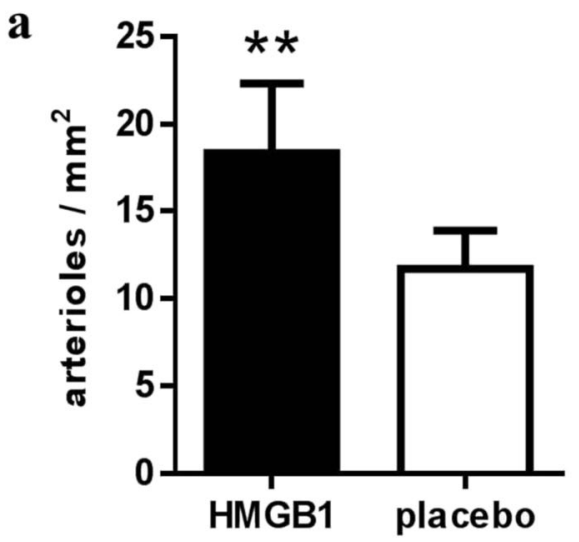

b

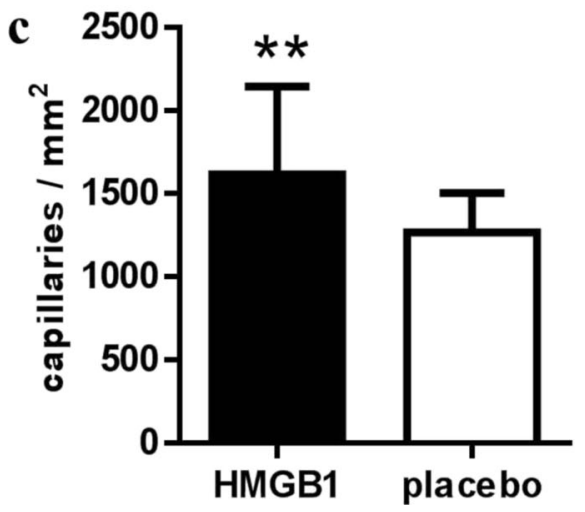

Fig. 5 Microvascular density at 60 days post-AMI. Arteriolar density (a) and capillary density (c) were higher in HMGB1-injected animals. b Representative slices of the injected peri-infarct region for each group showing arterioles stained with an antibody against smooth muscle actin

d
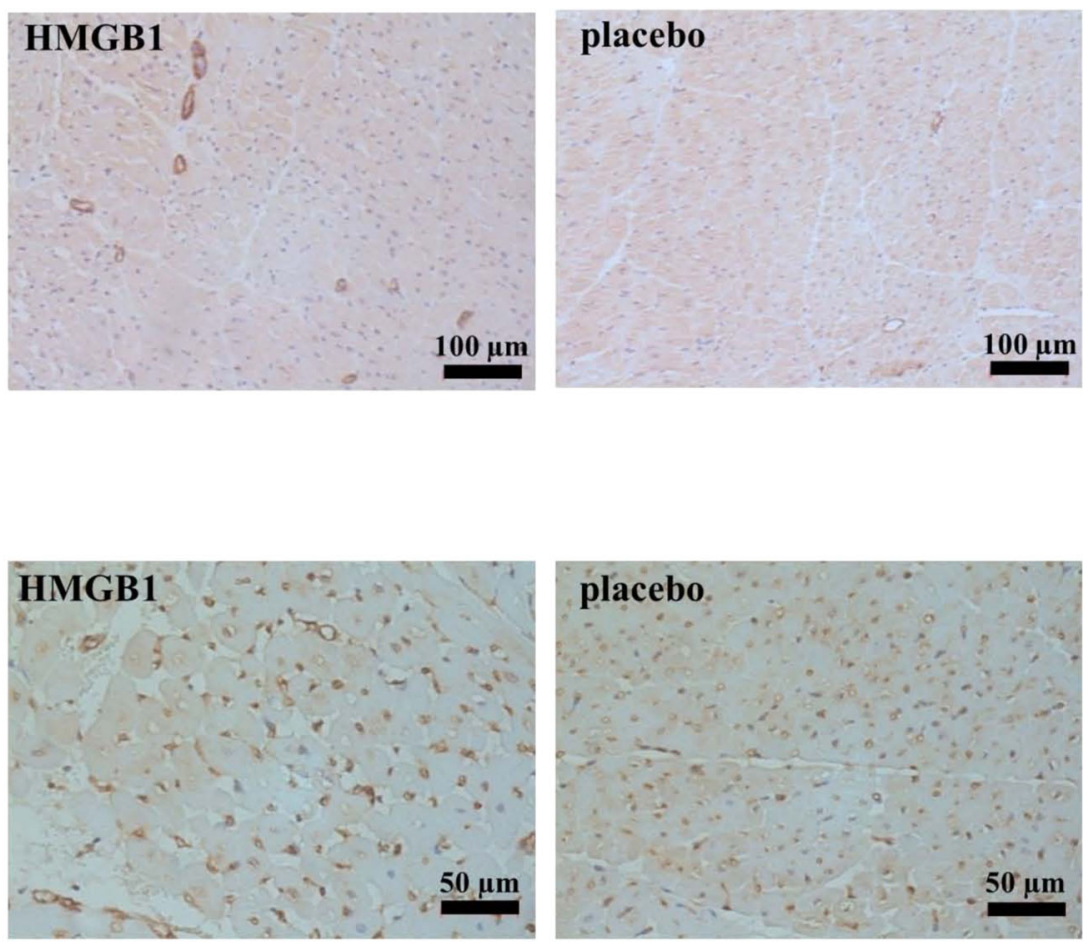

(bars $=100 \mu \mathrm{m}$ ). $\mathbf{d}$ Representative slices of the injected peri-infarct region for each group showing capillaries stained with a biotinylated lectin (bars $=50 \mu \mathrm{m}$ ). Results are mean $\pm \mathrm{SD}$. $* * P<0.01$ vs. placebo 
Table 2 Relative mRNA expression of angiogenic and cardiomyogenic genes at 60 days post-AMI

\begin{tabular}{llllll}
\hline & VEGF & Tbx20 & Ckit & Nkx2.5 & Gata4 \\
\hline HMGB1 & $0.97 \pm 0.86$ & $0.75 \pm 0.14$ & $1.00 \pm 0.38$ & $0.99 \pm 0.41$ & $1.15 \pm 0.75$ \\
Placebo & $1.18 \pm 0.91$ & $1.04 \pm 0.34$ & $1.21 \pm 0.89$ & $1.05 \pm 0.35$ & $1.14 \pm 0.63$ \\
\hline
\end{tabular}

$H M G B 1$, high mobility group box 1 protein. Data are means \pm SD. $n=6$ for all groups. No significant differences existed between groups administering a pro-inflammatory agent in this context may increase the electrical instability and thus the risk of ventricular fibrillation. The strategy of injecting the treatments $4 \mathrm{~h}$ after AMI has been applied before by other authors [15, 26].

The doses used for the dose-response protocol were extrapolated from previous studies in rodents with AMI, and adapted to the body weight of our sheep [13-15, 26]. First, we determined with a short-term follow-up protocol which of the two HMGB1 doses exerted cardioprotection in the animal model that would later be used to assess long-term effects on infarct size and LV function. Specifically, we aimed at assessing microvascular proliferation (capillary and arteriolar densities) and the presence of signs associated with cardiomyogenesis (cycling adult cardiomyocytes and expression of genes associated with recruitment of cardiac progenitor cells). We used a 1-week protocol because these events have been shown to be detectable at early time points after treatment [26]. Later on, it may be possible to observe the effect on cardioprotection, but not always the early processes leading to it.

To evaluate LV function, we used 3 time points. The first time point was set at 3 days after AMI, because we considered it appropriate to minimize the risks associated with postoperative instability and, at the same time, to be consistent with a control post-occlusion condition, on account that at that time the effects of the treatment would be minimal or undetectable. The final time point (60 days) exceeds (and even duplicates) that of prior studies addressing HMGB1 administration in other animal models of AMI $[12,14,15,26]$, and the mid-follow-up time point (30 days) was selected to observe if the changes followed a particular time course.

As it has been shown in mice with AMI [12, 13, 27], in our large mammalian model, HMGB1 induced angioarteriogenesis. Capillary growth was significantly higher than in placebo animals only in the group receiving the high dose of HMGB1. Arteriolar density, on the other hand, was numerically equal in animals receiving the high and the low HMGB1 doses. The lower data dispersion in the former explained that the difference with the placebo group achieved statistical significance. Arteriolar proliferation induced by angiogenic treatments is important because newly formed vessels displaying a smooth muscle layer respond to the physiological control of vascular tone, regulating tissue perfusion and actively participating in coronary autoregulation [28]. On the other hand, the vessels that directly interact with the cardiomyocytes in gas and metabolite exchange are the capillaries [29]. It is therefore sound to assume that, at least in part, the myocardial salvage induced by HMGB1 resulted from improved perfusion of the myocardium at risk.

Microvessels (especially capillaries) induced by angiogenic treatments have been shown to be unstable, tending to regress in the long term if there is no blood flowing in them [30-32]. This may explain why the absolute number of capillaries found in the short-term protocol was higher than that found at 60 days post-treatment. However, still at this time point, our HMGB1-treated sheep had significantly higher arteriolar and capillary densities than placebo-treated animals. HMGB1 has been reported to be a proangiogenic cytokine given its potent effect on endothelial cell sprouting [33]. The microvessels proliferation observed in our sheep included both capillaries and arterioles. This response can be attributed, at least in part, to $V E G F$ overexpression, on account that, in addition to inducing proliferation of vessels composed by a single layer of endothelial cells, VEGF has been reported to promote arteriogenesis in mice and pigs [20,34].

Another mechanism that likely contributed to infarct size reduction was cardiomyogenesis. In agreement with previous reports [25], 1 week after treatment, myocardial samples from animals receiving the high dose of HMGB1 showed approximately 5 adult myocyte nuclei per $\mathrm{mm}^{2}$ with evidence of cell cycling. From our data, it is not possible to know if eventually cytokinesis occurred, but it is to be noticed that the number of cycling myocytes was about 15-fold higher in HMGB1 than in placebotreated sheep.

Consistent with this observation, Tbx20, Nkx2.5, and Gata4 were overexpressed in animals treated with highdose HMGB1. Tbx20, a T-box cardiac transcription factor, is known to interact not only with $N k \times 2.5$ and Gata4 in the regulation of heart development [35] but also with genes required for $\mathrm{G} 1 / \mathrm{S}$ transition in the fetal cardiomyocyte cell cycle [36], which is consistent with the higher density of cycling myocytes in our HMGB1-treated sheep. Furthermore, along with Tbx20, Nkx2.5, and Gata4, Ckit was overexpressed, which strongly suggests recruitment of cardiac progenitor cells. This confirms in a large mammalian model of AMI an effect previously reported in mice $[26,37]$. 
Further mechanisms contributing to infarct size reduction in mice, like angiogenesis from bone marrow-derived endothelial progenitor cells [27] or attenuation of apoptosis and AMP-activated protein kinase-dependent autophagy [38] were not assessed in the present study.

In conflict with our results, it has been recently reported that HMGB1 inhibition or blockade induces post-AMI cardioprotection. Zhang et al. reported that in adult rats, intragastric administration of the antiretroviral protease inhibitor atazanavir attenuated post-AMI remodeling and preserved systolic performance likely due to blocking of myocardial inflammatory cytokines through an HMGB1/TLR 9 signaling pathway [18]. On the other hand, Qi et al. showed that in mice with myocardial infarction, overexpression of angiotensinconverting enzyme 2 (ACE2) reduced infarct size and preserved cardiac function, and that these effects correlated with downregulation of HMGB 1 and downstream proinflammatory cascades [17]. However, other concomitant mechanisms not necessarily correlated with HMGB1 inhibition may have contributed to the observed effects. For example, ACE2 increased the expression of the angiotensin II type 2 receptor (AT2R), which is known to antagonize the angiotensin II type 1 receptor (AT1R), thus contributing to the observed cardioprotection through vasorelaxation, natriuresis, and anti-fibrosis [39], independently from any inhibitory effect on HMGB1.

The angiogenic and cardiomyogenic effects of HMGB1 induced a significantly better post-AMI recovery of LV function in the long term as compared with placebo. This was particularly noticeable in regional mechanics. In effect, while in HMGB1 wall thickening values recovered to almost their pre-AMI levels, they remained low throughout the follow-up in placebo-treated animals. Global LV function, on the other hand, showed similar values at end of follow-up in both groups. However, the extent of recovery with regard to early post-AMI values at both 30 and 60 days achieved statistical significance only in the HMGB1 group. On account that the infarcts were not as large as to induce significant ventricular remodeling, it is sound to assume that the improvements displayed in regional mechanics were not reflected to the same extent in terms of global LV performance. In fact, superior increases in \%EF induced by HMGB1 have been reported in mice and rats with larger infarcts $[14,26]$.

Overall, our results suggest that, at the dose used, HMGB1 is beneficial. Importantly, the large animal model used, whose cardiac structure, size, and physiology resemble that of the human, eases the extrapolation of the results to the clinic. To be noted, unlike other regenerative strategies as, for example, stem cell therapy or gene transfer, human recombinant proteins exert rapid and controlled effects, which not only shorten the time elapsed between application and therapeutic effect, but also increase the level of safety.

\section{Conclusions}

Our results indicate that high-dose HMGB1 affords long-term cardioprotection in sheep with permanent coronary occlusion, confirming previous results in rodents and extending them to a large mammal more closely resembling the human. This effect consisted of reduced infarct size and improved LV function, associated with HMGB1-induced capillary and arteriolar proliferation and cardiomyogenesis. Further studies on larger infarcts inducing left ventricular remodeling and at longer follow-up periods are needed to evaluate the potential usefulness of this treatment in the clinical setting.

Acknowledgments We thank veterinarians María Inés Besansón and Pedro Iguain for anesthetic management and animal house assistants Juan Carlos Mansilla, Osvaldo Sosa, and Juan Ocampo for dedicated care of the animals. We also thank Julio Martínez, Fabián Gauna, and Rosana Valverdi for technical help.

Funding information Supported by grant 2012-1729 from the National Agency for the Promotion of Science and Technology (ANPCyT), Ministry of Science, Technology and Innovative Production (MINCyT) of Argentina.

\section{Compliance with ethical standards}

Conflict of interest The authors declare that they have no conflict of interest.

Ethical standards All procedures were carried out in accordance with the Guide for Care and Use of Laboratory Animals, published by the US National Institutes of Health (NIH Publication No. 85-23, revised 1996) and approved and monitored by the Laboratory Animal Care and Use Committee (CICUAL) of the Favaloro University (approval \# DCT015712). The manuscript does not contain clinical studies or patient data.

Publisher's note Springer Nature remains neutral with regard to jurisdictional claims in published maps and institutional affiliations.

\section{References}

1. Roth GA, Johnson C, Abajobir A, Abd-Allah F, Abera SF, Abyu G, et al. Global, regional, and national burden of cardiovascular diseases for 10 causes, 1990 to 2015. J Am Coll Cardiol. 2017;70:1-25.

2. Tiyyagura SR, Pinney SP. Left ventricular remodeling after myocardial infarction: past, present, and future. Mt Sinai J Med. 2006;73:840-51.

3. Stone GW, Selker HP, Thiele H, Patel MR, Udelson JE, Ohman $\mathrm{EM}$, et al. Relationship between infarct size and outcomes following primary PCI: patient-level analysis from 10 randomized trials. J Am Coll Cardiol. 2016;67:1674-83.

4. Gnavi R, Rusciani R, Dalmasso M, Giammaria M, Anselmino M, Roggeri DP, et al. Gender, socioeconomic position, revascularization procedures and mortality in patients presenting with STEMI and NSTEMI in the era of primary PCI. Differences or inequities? Int J Cardiol. 2014;176:724-30.

5. De Luca G, Petrelli A, Landriscina T, Gnavi R, Giammaria M, Costa G. Geographic and socioeconomic differences in access to 
revascularization following acute myocardial infarction. Eur J Pub Health. 2016;26:760-5.

6. Rebouças JS, Santos-Magalhães NS, Formiga FR. Cardiac regeneration using growth factors: advances and challenges. Arq Bras Cardiol. 2016;107:271-5.

7. Awada HK, Hwang MP, Wang Y. Towards comprehensive cardiac repair and regeneration after myocardial infarction: aspects to consider and proteins to deliver. Biomaterials. 2016;82:94-112.

8. Guo M, Shi JH, Wang PL, Shi DZ. Angiogenic growth factors for coronary artery disease: current status and prospects. J Cardiovasc Pharmacol Ther. 2018;23:130-41.

9. Bianchi ME, Agresti A. HMG proteins: dynamic players in gene regulation and differentiation. Curr Opin Genet Dev. 2005;15:496-506.

10. Celona B, Weiner A, Di Felice F, Mancuso FM, Cesarini E, Rossi RL, et al. Substantial histone reduction modulates genomewide nucleosomal occupancy and global transcriptional output. PLoS Biol. 2011;9:e1001086. https://doi.org/10.1371/journal.pbio. 1001086

11. Raucci A, Di Maggio S, Scavello F, D’Ambrosio A, Bianchi M, Capogrossi MC. The Janus face of HMGB1 in heart disease: a necessary update. Cell Mol Life Sci. 2019;76:211-29.

12. Kitahara T, Takeishi Y, Harada M, Niizeki T, Suzuki S, Sasaki T, et al. Highmobility group box 1 restores cardiac function after myocardial infarction in transgenic mice. Cardiovasc Res. 2008;80:40-6.

13. Limana F, Esposito G, D'Arcangelo D, Di Carlo A, Romani S, Melillo G, et al. HMGB1 attenuates cardiac remodelling in the failing heart via enhanced cardiac regeneration and mir-206- mediated inhibition of timp-3. PLoS One. 2011;6(6):e19845. https://doi. org/10.1371/journal.pone.0019845.

14. Takahashi K, Fukushima S, Yamahara K, Yashiro K, Shintani Y, Coppen SR, et al. Modulated inflammation by injection of highmobility group box 1 recovers post-infarction chronically failing heart. Circulation. 2008;118(Suppl 1):S106-14.

15. Zhou X, Hu X, Xie J, Xu C, Xu W, Jiang H. Exogenous highmobility group box 1 protein injection improves cardiac function after myocardial infarction: involvement of Wnt signaling activation. J Biomed Biotechnol. 2012;2012:1-5. https://doi.org/10.1155/ 2012/743879.

16. Di Maggio S, Milano G, De Marchis F, D'Ambrosio A, Bertolotti $\mathrm{M}$, Palacios BS, et al. Non-oxidizable HMGB1 induces cardiac fibroblasts migration via CXCR4 in a CXCL12-independent manner and worsens tissue remodeling after myocardial infarction. Biochim Biophys Acta Mol basis Dis. 2017;1863:2693-704.

17. Qi YF, Zhang J, Wang L, Shenoy V, Krause E, Oh SP, et al. Angiotensin-converting enzyme 2 inhibits high-mobility group box 1 and attenuates cardiac dysfunction post-myocardial ischemia. J Mol Med. 2016;94:37-49.

18. Zhang G, Zhang X, Li D, Tian J, Jiang W. Long-term oral atazanavir attenuates myocardial infarction-induced cardiac fibrosis. Eur J Pharmacol. 2018;828:97-102.

19. Locatelli P, Olea FD, Mendiz O, Salmo F, Fazzi L, Hnatiuk A, et al. An ovine model of postinfarction dilated cardiomyopathy in animals with highly variable coronary anatomy. ILAR J. 2011;52: E16-21.

20. Crottogini A, Meckert PC, Vera Janavel G, Lascano E, Negroni J, Del Valle H, et al. Arteriogenesis induced by intramyocardial vascular endothelial growth factor 165 gene transfer in chronically ischemic pigs. Hum Gene Ther. 2003;14:1307-18.

21. Laguens R, Cabeza Meckert P, Vera Janavel G, De Lorenzi A, Lascano E, Negroni J, et al. Cardiomyocyte hyperplasia after plasmid-mediated vascular endothelial growth factor gene transfer in pigs with chronic myocardial ischemia. J Gene Med. 2004;6: 222-7.

22. Locatelli P, Olea FD, De Lorenzi A, Salmo F, Vera Janavel GL, Hnatiuk AP, et al. Reference values for echocardiographic parameters and indexes of left ventricular function in healthy, young adult sheep used in translational research: comparison with standardized values in humans. Int J Clin Exp Med. 2011;4:258-64.

23. Gräbner W, Pfitzer P. Number of nuclei in isolated myocardial cells of pigs. Virchows Arch B Cell Pathol. 1974;15:279-94.

24. Adler CP, Friedburg H, Herget GW, Neuburger M, Schwalb H. Variability of cardiomyocyte DNA content, ploidy level and nuclear number in mammalian hearts. Virchows Arch. 1996;429:159-64.

25. de Haan JJ, Smeets MB, Pasterkamp G, Arslan F. Danger signals in the initiation of the inflammatory response after myocardial infarction. Mediat Inflamm. 2013;2013:1-13. https://doi.org/10.1155/ 2013/206039.

26. Limana F, Germani A, Zacheo A, Kajstura J, Di Carlo A, Borsellino $\mathrm{G}$, et al. Exogenous high-mobility group box 1 protein induces myocardial regeneration after infarction via enhanced cardiac C-kit+ cell proliferation and differentiation. Circ Res. 2005;97:e73-83.

27. Nakamura Y, Suzuki S, Shimizu T, Miyata M, Shishido T, Ikeda K, et al. High mobility group box 1 promotes angiogenesis from bone marrow-derived endothelial progenitor cells after myocardial infarction. J Atheroscler Thromb. 2015;22:570-81.

28. Westerhof N, Boer C, Lamberts R, Sipkema P. Cross-talk between cardiac muscle and coronary vasculature. Physiol Rev. 2006;86: 1263-308.

29. Opie LH. Oxygen supply: coronary flow. In: Opie LH, editor. The heart. Physiology and metabolism. 2nd ed. New York: Raven Press; 1991. p. 277-81.

30. Tang K, Breen EC, Gerber HP, Ferrara NM, Wagner PD. Capillary regression in vascular endothelial growth factor-deficient skeletal muscle. Physiol Genomics. 2004;18:63-9.

31. Gounis MJ, Spiga MG, Graham RM, Wilson A, Haliko S, Lieber $\mathrm{BB}$, et al. Angiogenesis is confined to the transient period of VEGF expression that follows adenoviral gene delivery to ischemic muscle. Gene Ther. 2005;12:762-71.

32. Olea FD, Vera Janavel G, Cuniberti L, Yannarelli G, Cabeza Meckert P, Cors J, et al. Repeated, but not single, VEGF gene transfer affords protection against ischemic muscle lesions in rabbits with hindlimb ischemia. Gene Ther. 2009;16:716-23.

33. Mitola S, Belleri M, Urbinati C, Coltrini D, Sparatore B, Pedrazzi $\mathrm{M}$, et al. Cutting edge: extracellular high mobility group box-1 protein is a proangiogenic cytokine. J Immunol. 2006;176:12-5.

34. Clayton JA, Chalothorn D, Faber JE. Vascular endothelial growth factor-A specifies formation of native collaterals and regulates collateral growth in ischemia. Circ Res. 2008;103:1027-36.

35. Stennard FA, Costa MW, Elliott DA, Rankin S, Haast SJ, Lai D, et al. Cardiac T-box factor Tbx20 directly interacts with Nkx2-5, GATA4, and GATA5 in regulation of gene expression in the developing heart. Dev Biol. 2003;262:206-24.

36. Boogerd CJ, Zhu X, Aneas I, Sakabe NJ, Zhang L, Sobreira DR, et al. Tbx20 is required in mid-gestation cardiomyocytes and plays a central role in atrial development. Circ Res. 2018. https://doi.org/ 10.1161/CIRCRESAHA.118.311339.

37. Germani A, Limana F, Capogrossi MC. Pivotal advances: highmobility group box 1 protein - a cytokine with a role in cardiac repair. J Leukoc Biol. 2007;81:41-5.

38. Foglio E, Puddighinu G, Germani A, Russo MA, Limana F. HMGB1 inhibits apoptosis following MI and induces autophagy via mTORC1 inhibition. J Cell Physiol. 2017;232:1135-43.

39. AbdAlla S, Lother H, Abdel-tawab AM, Quitterer U. The angiotensin II AT2 receptor is an AT1 receptor antagonist. J Biol Chem. 2001;276:39721-6. 